\title{
Respiratory Reviews in Asthma 2013
}

\section{Tae-Hyung Kim, M.D.}

Department of Internal Medicine, Hanyang University Guri Hospital, Hanyang University College of

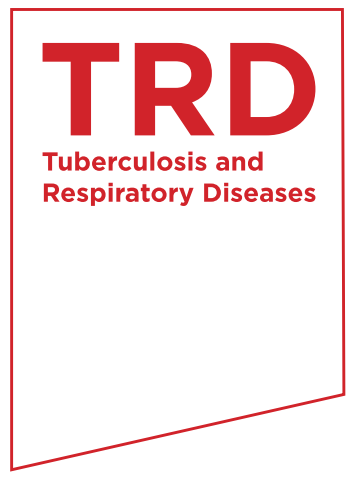
Medicine, Seoul, Korea

From January 2012 up until March 2013, many articles with huge clinical importance in asthma were published based on large numbered clinical trials or meta-analysis. The main subjects of these studies were the new therapeutic plan based on the asthma phenotype or efficacy along with the safety issues regarding the current treatment guidelines. For efficacy and safety issues, inhaled corticosteroid tapering strategy or continued long-acting beta agonists use was the major concern. As new therapeutic trials, monoclonal antibodies or macrolide antibiotics based on inflammatory phenotypes have been under investigation, with promising preliminary results. There were other issues on the disease susceptibility or genetic background of asthma, particularly for the "severe asthma" phenotype. In the era of genome and pharmacogenetics, there have been extensive studies to identify susceptible candidate genes based on the results of genome wide association studies (GWAS). However, for severe asthma, which is where most of the mortality or medical costs develop, it is very unclear. Moreover, there have been some efforts to find important genetic information in order to predict the possible disease progression, but with few significant results up until now. In conclusion, there are new ongoing aspects in the phenotypic classification of asthma and therapeutic strategy according to the phenotypic variations. With more pharmacogenomic information and clear identification of the "severe asthma" group even before disease progression from GWAS data, more adequate and individualized therapeutic strategy could be realized in the future.

Keywords: Asthma; Phenotype; Pharmacogenetics; Therapeutics

\section{Selection of Articles}

Among the articles dealing with clinical aspects of adult asthma published between January 2012 and March 2013, several important articles in the aspect of clinical manifestations and treatment were selected. Main subject journals were

Address for correspondence: Tae-Hyung Kim, M.D.

Department of Internal Medicine, Hanyang University Guri Hospital, Hanyang University College of Medicine, 153 Gyeongchun-ro, Guri 471701, Korea

Phone: 82-31-560-2240, Fax: 82-31-553-7369

E-mail: drterry@hanyang.ac.kr

Received: Feb. 16, 2014

Revised: Feb. 21, 2014

Accepted: Feb. 28, 2014

(c) It is identical to the Creative Commons Attribution Non-Commercial License (http://creativecommons.org/licenses/by-nc/3.0/).

Copyright ( 2014

The Korean Academy of Tuberculosis and Respiratory Diseases.

All rights reserved. as follows: American Journal of Respiratory and Critical Care Medicine, Thorax, Chest, European Respiratory Journal, New England Journal of Medicine, JAMA, Lancet, and Journal of Allergy and Clinical Immunology. We would introduce the articles in the order of new therapeutic agents, asthma control, diagnosis, severity systems and recent genome-wide association studies (GWAS).

\section{Treatment}

\section{New therapeutic agents}

Recently, various study results about the effect of new biochemical treatment according to the inflammatory phenotype of asthma like Omalizumab (anti-IgE Ab), Mepolizumab (antiIL $5 \mathrm{Ab}$ ), and Lebrikizumab (anti-IL $13 \mathrm{Ab}$ ) have been published. Furthermore, treatment effect and possible indications of tiotropium or macrolides in asthma have been investigated. 
Mepolizumab for severe eosinophilic asthma (DREAM): a multicentre, double-blind, placebo-controlled trial. Pavord et al. ${ }^{1}$ Lancet 2012;380:651-9.

Background: Some patients with severe asthma have recurrent asthma exacerbations associated with eosinophilic airway infl ammation. Early studies suggest that inhibition of eosinophilic airway inflammation with mepolizumab-a monoclonal antibody against interleukin 5-is associated with a reduced risk of exacerbations. We aimed to establish effi cacy, safety, and patient characteristics associated with the response to mepolizumab.

Methods: We undertook a multicentre, double-blind, placebocontrolled trial at 81 centres in 13 countries between Nov 9, 2009, and Dec 5, 2011. Eligible patients were aged 12-74 years, had a history of recurrent severe asthma exacerbations, and had signs of eosinophilic inflammation. They were randomly assigned (in a 1:1:1:1 ratio) to receive one of three doses of intravenous mepolizumab (75 mg, $250 \mathrm{mg}$, or $750 \mathrm{mg}$ ) or matched placebo $(100 \mathrm{~mL} 0.9 \% \mathrm{NaCl})$ with a central telephone-based system and computer-generated randomly permuted block schedule stratified by whether treatment with oral corticosteroids was required. Patients received 13 infusions at 4-week intervals. The primary outcome was the rate of clinically signifi cant asthma exacerbations, which were defi ned as validated episodes of acute asthma requiring treatment with oral corticosteroids, admission, or a visit to an emergency department. Patients, clinicians, and data analysts were masked to treatment assignment. Analyses were by intention to treat. This trial is registered with ClinicalTrials.gov, number NCT01000506.

Findings: 621 patients were randomised: 159 were assigned to placebo, 154 to $75 \mathrm{mg}$ mepolizumab, 152 to $250 \mathrm{mg}$ mepolizumab, and 156 to $750 \mathrm{mg}$ mepolizumab. 776 exacerbations were deemed to be clinically signifi cant. The rate of clinically signifi cant exacerbations was 2.40 per patient per year in the placebo group, 1.24 in the $75 \mathrm{mg}$ mepolizumab group (48\% reduction, 95\% CI 31-61\%; $\mathrm{p}<0.0001), 1.46$ in the $250 \mathrm{mg}$ mepolizumab group (39\% reduction, $19-54 \%$; $\mathrm{p}=0.0005$ ), and 1.15 in the $750 \mathrm{mg}$ mepolizumab group (52\% reduction, $36-64 \%$; $\mathrm{p}<0.0001)$. Three patients died during the study, but the deaths were not deemed to be related to treatment.

Interpretation: Mepolizumab is an eff ective and well tolerated treatment that reduces the risk of asthma exacerbations in patients with severe eosinophilic asthma. (Parvord et al. ${ }^{1}, 2012, p$. 651; Reprinted with permission of Elsevier Ltd.)

1) Comments: The authors suggested that mepolizumab as an effective and safe option which could reduce exacerbations in the subgroup of severe asthma with eosinophilic phenotype. Treatment options based on phenotypic variation have been tried due to the observed individual variation in the aspect of treatment response. For the effective biomarkers symbolizing the "eosinophilic inflammation," previous studies ${ }^{2}$ used sputum eosinophils, serum IgE, or another some biomarkers like interleukin (IL)-4, -5, or -13. In this study, exhaled nitric oxide concentration $\left(\mathrm{FE}_{\mathrm{NO}}\right)$, peripheral blood eosinophil count, or prompt deterioration of asthma control after a $25 \%$ or less reduction in inhaled corticosteroid (ICS) or oral corticosteroids in addition to the sputum eosinophil count were used. These composite indices for eosinophilic inflammation could be more practical than previous biomarkers. Another issue is the dose-response effect of mepolizumab on exacerbations. Previous study with mepolizumab ${ }^{2}$ was performed with $750 \mathrm{mg}$ mepolizumab. In this study, even the dose of $75 \mathrm{mg}$ which is 10 times lower was also effective. The dose-response relation between mepolizumab and eosinophils in blood and sputum suggests that the lowest dose also is near to the top of the dose-response curve.

\section{Azithromycin for prevention of exacerbations in severe asthma (AZISAST): a multicentre randomised double- blind placebo-controlled trial. Brusselle et al. ${ }^{3}$ Thorax 2013;68:322-9.}

Background: Patients with severe asthma are at increased risk of exacerbations and lower respiratory tract infections (LRTI). Severe asthma is heterogeneous, encompassing eosinophilic and non-eosinophilic (mainly neutrophilic) phenotypes. Patients with neutropilic airway diseases may benefit from macrolides.

Methods: We performed a randomised double-blind placebocontrolled trial in subjects with exacerbationprone severe asthma. Subjects received low-dose azithromycin $(n=55)$ or placebo $(n=54)$ as add-on treatment to combination therapy of inhaled corticosteroids and long-acting $\beta 2$ agonists for 6 months. The primary outcome was the rate of severe exacerbations and LRTI requiring treatment with antibiotics during the 26-week treatment phase. Secondary efficacy outcomes included lung function and scores on the Asthma Control Questionnaire (ACQ) and Asthma Quality of Life Questionnaire (AQLQ). Results: The rate of primary endpoints (PEPs) during 6 months was not significantly different between the two treatment groups: 0.75 PEPs (95\% CI 0.55 to 1.01) per subject in the azithromycin group versus 0.81 PEPs (95\% CI 0.61 to 1.09) in the placebo group $(\mathrm{p}=0.682)$. In a predefined subgroup analysis according to the inflammatory phenotype, azithromycin was associated with a significantly lower PEP rate than placebo in subjects with noneosinophilic severe asthma (blood eosinophilia $\leq 200 / \mu \mathrm{l}$ ): 0.44 PEPs (95\% CI 0.25 to 0.78 ) versus 1.03 PEPs (95\% CI 0.72 to 1.48 ) ( $\mathrm{p}=0.013)$. Azithromycin significantly improved the AQLQ score but there were no significant between-group differences in the ACQ score or lung function. Azithromycin was well tolerated, but was associated with increased oropharyngeal carriage of macrolide-resistant streptococci.

Conclusions: Azithromycin did not reduce the rate of severe 
exacerbations and LRTI in patients with severe asthma. However, the significant reduction in the PEP rate in azithromycintreated patients with non-eosinophilic severe asthma warrants further study. (Brusselle et al. ${ }^{3}$, p. 322; Reprinted with permission of BMJ Publishing Group Ltd.)

2) Comments: There are significant proportion with noneosinophilic inflammatory phenotype in severe asthma population, and based on some evidence of macrolide effect on neutrophilic airway inflammatory diseases like bronchiectasis or chronic obstructive pulmonary disease (COPD) ${ }^{4}$, azithromycin could be considered in non-eosinophilic severe asthma subgroup. Previous study with short term clarithromycin treatment in addition to ICS ${ }^{5}$ in suboptimally controlled mildto-moderate persistent asthma showed slight improvement in airway hyperresponsiveness, but failed to show improvements in asthma control, lung function or another secondary outcomes. In this study, even though azithromycin did not reduce severe exacerbations and lower respiratory tract infections (LRTI) in all patients with severe asthma, the authors claimed that the significant reduction in the primary endpoint rate in azithromycin-treated patients with non-eosinophilic severe asthma warrants further study. Also, there are another issues of long term azithromycin treatment: cost effectiveness and predictable side effect-gastroinstestinal upset and colonization of resistant bacteria. In this study, azithromycin was well tolerated and increased oropharyngeal colonization did not result in the increased LRTI or pneumonia. Overally, azithromycin could be safe and effective option for severe, non-eosinophilic asthma with frequent exacerbation. But, there should be another concern for the long-term effects of macrolide treatment on community-based microbial resistance.

\section{Tiotropium in asthma poorly controlled with standard combination therapy. Kerstjens et al. ${ }^{6} \mathrm{~N}$ Engl J Med 2012;367:1198-207.}

Background: Some patients with asthma have frequent exacerbations and persistent airflow obstruction despite treatment with inhaled glucocorticoids and long-acting beta-agonists (LABAs).

Methods: In two replicate, randomized, controlled trials involving 912 patients with asthma who were receiving inhaled glucocorticoids and LABAs, we compared the effect on lung function and exacerbations of adding tiotropium (a total dose of 5 $\mu \mathrm{g}$ ) or placebo, both delivered by a soft-mist inhaler once daily for 48 weeks. All the patients were symptomatic, had a postbronchodilator forced expiratory volume in 1 second (FEV1) of $80 \%$ or less of the predicted value, and had a history of at least one severe exacerbation in the previous year.

Results: The patients had a mean baseline FEV1 of $62 \%$ of the predicted value; the mean age was 53 years. At 24 weeks, the mean $( \pm \mathrm{SE})$ change in the peak FEV1 from baseline was greater with tiotropium than with placebo in the two trials: a difference of $86 \pm 34 \mathrm{ml}$ in trial $1(\mathrm{P}=0.01)$ and $154 \pm 32 \mathrm{ml}$ in trial $2(\mathrm{P}<0.001)$. The predose (trough) FEV1 also improved in trials 1 and 2 with tiotropium, as compared with placebo: a difference of $88 \pm 31 \mathrm{ml}$ $(\mathrm{P}=0.01)$ and $111 \pm 30 \mathrm{ml}(\mathrm{P}<0.001)$, respectively. The addition of tiotropium increased the time to the first severe exacerbation (282 days vs. 226 days), with an overall reduction of $21 \%$ in the risk of a severe exacerbation (hazard ratio, $0.79 ; \mathrm{P}=0.03$ ). No deaths occurred; adverse events were similar in the two groups. Conclusions: In patients with poorly controlled asthma despite the use of inhaled glucocorticoids and LABAs, the addition of tiotropium significantly increased the time to the first severe exacerbation and provided modest sustained bronchodilation. (Kerstjens et al. ${ }^{6}$, p. 1198; Reprinted with permission of Massachusetts Medical Society)

3) Comments: This study showed that in the patients with poorly controlled asthma despite the use of ICS and long-acting beta-agonists (LABAs), the addition of tiotropium could be another effective therapeutic option. Tiotropium addition significantly increased the time to the first severe exacerbation and provided modest sustained bronchodilation. Antiinflammatory treatment with mainly ICS was the main stream treatment in asthma. But, in the poorly controlled asthma, add on therapy with combination treatment including bronchodilator-mainly LABA and to a less extent, theophylline-or another anti-inflammatory treatments including leukotriene receptor antagonists, or special medication based on inflammatory phenotype-monoclonal $\mathrm{Ab}$ for IgE, IL-4/13, macrolide etc.-could be another treatment options. Tiotropium is well established, most widely used long acting bronchodilator in COPD and its safety profile was also well studied. In this study, the effect and safety of tiotropium was effectively studied in large sized population. Therefore, tiotropium should be considered in the patients with severe, poorly controlled asthma with ICS and LABA treatment.

\section{Change of treatment steps according to the asthma con- trol status}

Theoretical background for step-up or step-down therapy according to the international guideline of asthma lies on the degree of asthma control. In order to make more appropriate estimation of asthma control, repetitive measurement of specific biomarkers and patient-recorded asthma symptom diary are currently in use in addition to the physician assessment of symptom changes and lung function. And, there are studies comparing the relative efficacy of those measurements.

Furthermore, there are studies about the role of LABA in the treatment of asthma when the principle of step-down therapy is applicated in the aspect of the efficacy and cardiovascular risk. 
Comparison of physician-, biomarker-, and symptombased strategies for adjustment of inhaled corticosteroid therapy in adults with asthma: the BASALT randomized controlled trial. Calhoun et al. ${ }^{7}$ JAMA 2012;308:987-97.

Context: No consensus exists for adjusting inhaled corticosteroid therapy in patients with asthma. Approaches include adjustment at outpatient visits guided by physician assessment of asthma control (symptoms, rescue therapy, pulmonary function), based on exhaled nitric oxide, or on a day-to-day basis guided by symptoms.

Objective: To determine if adjustment of inhaled corticosteroid therapy based on exhaled nitric oxide or day-to-day symptoms is superior to guideline-informed, physician assessment-based adjustment in preventing treatment failure in adults with mild to moderate asthma.

Design, Setting, and Participants: A randomized, parallel, 3-group, placebocontrolled, multiply-blinded trial of 342 adults with mild to moderate asthma controlled by low-dose inhaled corticosteroid therapy ( $\mathrm{n}=114$ assigned to physician assessment-based adjustment [101 completed], n=115 to biomarkerbased [exhaled nitric oxide] adjustment [92 completed], and $\mathrm{n}=113$ to symptom-based adjustment [97 completed]), the Best Adjustment Strategy for Asthma in the Long Term (BASALT) trial was conducted by the Asthma Clinical Research Network at 10 academic medical centers in the United States for 9 months between June 2007 and July 2010.

Interventions: For physician assessment-based adjustment and biomarker-based (exhaled nitric oxide) adjustment, the dose of inhaled corticosteroids was adjusted every 6 weeks; for symptom-based adjustment, inhaled corticosteroids were taken with each albuterol rescue use.

Main Outcome Measure: The primary outcome was time to treatment failure.

Results: There were no significant differences in time to treatment failure. The 9-month Kaplan-Meier failure rates were 22\% (97.5\% CI, 14\%-33\%; 24 events) for physician assessment-based adjustment, 20\% (97.5\% CI, 13\%-30\%; 21 events) for biomarkerbased adjustment, and 15\% (97.5\% CI, 9\%-25\%; 16 events) for symptom-based adjustment. The hazard ratio for physician assessment-based adjustment vs biomarker based adjustment was 1.2 (97.5\% CI, 0.6-2.3). The hazard ratio for physician assessment-based adjustment vs symptom-based adjustment was 1.6 (97.5\% CI, 0.8-3.3).

Conclusion: Among adults with mild to moderate persistent asthma controlled with low-dose inhaled corticosteroid therapy, the use of either biomarker-based or symptom based adjustment of inhaled corticosteroids was not superior to physician assessment-based adjustment of inhaled corticosteroids in time to treatment failure. (Calhoun et al. ${ }^{7}$, p. 987; Reprinted with permission of American Medical Association)
1) Comments: Because either biomarker-based adjustment (BBA) or symptom-based adjustment (SBA) of ICS was not superior to physician assessment-based adjustment among adults with mild to moderate persistent asthma in time to treatment failure in this study, the authors concluded that more generalized application of BBA or SBA, in which additional costs or patient's efforts are needed, warrants more investigations. Biomarkers like sputum eosinophils, exhaled nitric oxide, or methacholine responsiveness have had limited practical aspects and caused higher cost. Even though SBA of ICS dose may be also appropriate in most patients with mild to moderate asthma, the perception of individual symptoms of asthma could be poor and variable among patients, resulting less favorable outcomes. Because of the small sample size, the results could not determine the associations of ethnicity and race with responsiveness to adjustment strategy. Therefore, a very large study could be needed to prove statistical significance for a difference in treatment failure.

Long-acting beta2-agonist step-off in patients with controlled asthma. Brozek et al. ${ }^{8}$ Arch Intern Med 2012;172:1365-75.

Background: Because of concerns about the safety of long-acting2-agonist (LABA) use in patients with asthma, withdrawal of the LABA is recommended by the US Food and Drug Administration once asthma is controlled by combination therapy with a LABA and inhaled corticosteroid (ICS).

Objective: To perform a systematic review and metaanalysis assessing evidence supporting the discontinuation of LABA therapy once asthma control has been achieved with a combination of ICS and LABA.

Data Sources: MEDLINE, EMBASE, and Cochrane Central Register of Controlled Trials databases were searched (through August 2010), references of identified studies and selected narrative review articleswere evaluated, registries of clinical trials were reviewed, and manufacturers of LABAs were contacted.

Study Selection: Randomized controlled trials of discontinuation of LABA therapy in patients with asthma controlled with a combination of ICS and LABA.

Results: Of 1492 screened articles, only 5 trials involving patients aged 15 years or older fulfilled a priori-specified inclusion criteria. Results did not favor the LABA step-off approach compared with no change in treatment. The LABA step-off regimen increased asthma impairment, with worse Asthma Quality of Life Questionnaire score (mean difference [95\% CI], 0.32 [0.140.51] points lower); worse Asthma Control Questionnaire score (0.24 [0.13-0.35] points higher); fewer symptom-free days (9.15\% [1.62\%-16.69\%] less); and greater risk of withdrawal from study resulting from lack of efficacy or loss of asthma control (risk ratio, 3.27 [2.16-4.96]). Risk of exacerbations and deaths after LABA step-off were not evaluable because of the small number of events and short duration of follow-up. 
Conclusions: Evidence suggests that discontinuing LABA therapy in adults and older children with asthma controlled with a combination of ICSs and LABAs results in increased asthma-associated impairment. Additional trials measuring all long-term patient-important outcomes are needed. (Brozek et $a l^{8}$, p. 1365; Reprinted with permission of American Medical Association)

2) Comments: Overall results suggested that discontinuation of LABA in adults and older children after asthma control by ICS-LABA combination may results in asthma-associated impairment. This is the first systematic review and meta-analysis of randomized controlled trial investigating the effect of LABA step-off in the patients with asthma controlled by ICSLABA combination therapy. The number of studies investigating this subject was much smaller than expected, the power of this analysis could be smaller and somewhat inaccurate. But, even from the limited data, discontinuation of LABA compared to continued use of ICS-LABA combination increased the risk of the loss of asthma control. There have been the safety issues or side effects like pneumonia risk or cardiovascular events for ICS or LABA, respectively. Therefore, for the maintenance therapy with controlled patients with long-term ICS-LABA combination, LABA step off or dose adjustment of ICS could be major concern and it needs consideration of individual risk factors associated with pneumonia or cardiovascular events. Also, additional investigations with long-term patient-important outcomes are needed.

The relationship between combination inhaled corticosteroid and long-acting beta-agonist use and severe asthma exacerbations in a diverse population. Wells et al. ${ }^{9} \mathrm{~J}$ Allergy Clin Immunol 2012;129:1274-9.

Background: Safety concerns surround the use of long-acting b-agonists (LABAs) for the treatment of asthma, even in combination with inhaled corticosteroids (ICSs) and particularly in high-risk subgroups.

Objective: To estimate the effect of ICS therapy and fixed-dose ICS/LABA combination therapy on severe asthma exacerbations in a racially diverse population.

Methods: ICS and ICS/LABA exposure was estimated from pharmacy data for patients with asthma aged 12 to 56 years who were members of a large health maintenance organization. ICS and ICS/LABA use was estimated for each day of follow-up to create a moving window of exposure. Proportional hazard models were used to assess the relationship between ICS and ICS/LABA combination therapy and severe asthma exacerbations (ie, use of oral corticosteroids, asthma-related emergency department visit, or asthma-related hospitalization).

Results: Among the 1828 patients who met the inclusion criteria, 37\% were African American, 46\% were treated with ICS therapy alone, and 54\% were treated with an ICS/LABA combination. Models assessing the risk of severe asthma exacerbations among individuals using ICS treatment alone and ICS/LABA combination therapy suggested that the overall protective effect was as good or better for ICS/LABA combination therapy when compared with ICS treatment alone (hazard ratio, 0.65 vs 0.72 , respectively). Analyses in several subgroups, including African American patients, showed a similar statistically significant protective association for combination therapy. Conclusion: Treatment with ICS/LABA fixed-dose combination therapy appeared to perform as well as or better than ICS treatment alone in reducing severe asthma exacerbations; this included multiple high-risk subgroups. (Wells et al. ${ }^{9}, p .1274$; Reprinted with permission of Elsevier Ltd.)

3) Comments: The authors proclaimed that fixed dose ICS-LABA therapy does not increase overall risk compared to ICS alone, and show similar protective effect on severe asthma exacerbation. Therefore, including LABA could be safe and important treatment regimen despite of previous reports of adverse outcome. This study has the strength of large sized population-based observational study and the first study to attempt to measure actual ICS and ICS-LABA exposure with regard to asthma exacerbations. But, this study also demonstrates that the beneficial effects of ICS-LABA combination therapy could be lowered if the dose exceeds the recommended level. Actually, using ICS alone could be more beneficial when compared with ICS-LABA that exceeds the recommended dose, probably from the side effects associated with LABA dose-up. There should be concerns for the individual risk factors for the common side effects like pneumonia or cardiovascular events.

\section{Diagnosis}

\section{Biomarkers}

Methacholine challenge test: diagnostic characteristics in asthmatic patients receiving controller medications. Sumino et al. ${ }^{10} \mathrm{~J}$ Allergy Clin Immunol 2012;130:69-75.

Background: The methacholine challenge test (MCT) is commonly used to assess airway hyperresponsiveness, but the diagnostic characteristics have not been well studied in asthmatic patients receiving controller medications after the use of highpotency inhaled corticosteroids became common.

Objectives: We investigated the ability of the MCT to differentiate participants with a physician's diagnosis of asthma from nonasthmatic participants.

Methods: We conducted a cohort-control study in asthmatic participants $(\mathrm{n}=126)$ who were receiving regular controller medications and nonasthmatic control participants $(n=93)$ to evaluate the sensitivity and specificity of the MCT. 
Results: The overall sensitivity was $77 \%$ and the specificity was 96\% with a threshold PC20 (the provocative concentration of methacholine that results in a 20\% drop in FEV1) of $8 \mathrm{mg} /$ $\mathrm{mL}$. The sensitivity was significantly lower in white than in African American participants (69\% vs 95\%, $\mathrm{P}=.015$ ) and higher in atopic compared with nonatopic ( $82 \%$ vs $52 \%, \mathrm{P}=.005)$. Increasing the PC20 threshold from 8 to $16 \mathrm{mg} / \mathrm{mL}$ did not noticeably improve the performance characteristics of the test. African American race, presence of atopy, and lower percent predicted FEV1 were associated with a positive test result.

Conclusions: The utility of the MCT to rule out a diagnosis of asthma depends on racial and atopic characteristics. Clinicians should take into account the reduced sensitivity of the MCT in white and nonatopic asthmatic patients when using this test for the diagnosis of asthma. (Sumino et al. ${ }^{10}$, p. 69; Reprinted with permission of Elsevier Ltd.)

1) Comments: This study showed that race and atopic status can influence the efficacy of the methacholine challenge test (MCT) to rule out asthma. Therefore, the authors claimed that clinicians must consider about reduced sensitivity of the MCT in Caucasian and non-atopic patients. Because this study does not include Asian population, the sensitivity of MCT in Asian could not be determined. Therefore, this study warrants similar study including Asian population. Recently, there have been also safety concerns and the costs issues for using industrial methacholine as the material of performing MCT in Korea, similar study for the sensitivity and specificity of mannitol for diagnosing asthma and the comparison study between methacholin and mannitol for diagnostic performance should be performed.

Periostin is a systemic biomarker of eosinophilic airway inflammation in asthmatic patients. Jia et al. ${ }^{11} \mathrm{~J} \mathrm{Al}$ lergy Clin Immunol 2012;130:647-54.

Background: Eosinophilic airway inflammation is heterogeneous in asthmatic patients. We recently described a distinct subtype of asthma defined by the expression of genes inducible by $\mathrm{T}_{\mathrm{H}} 2$ cytokines in bronchial epithelium. This gene signature, which includes periostin, is present in approximately half of asthmatic patients and correlates with eosinophilic airway inflammation. However, identification of this subtype depends on invasive airway sampling, and hence noninvasive biomarkers of this phenotype are desirable.

Objective: We sought to identify systemic biomarkers of eosinophilic airway inflammation in asthmatic patients.

Methods: We measured fraction of exhaled nitric oxide (FENO), peripheral blood eosinophil, periostin, YKL-40, and IgE levels and compared these biomarkers with airway eosinophilia in asthmatic patients.

Results: We collected sputum, performed bronchoscopy, and matched peripheral blood samples from 67 asthmatic pa- tients who remained symptomatic despite maximal inhaled corticosteroid treatment (mean FEV1, 60\% of predicted value; mean Asthma Control Questionnaire [ACQ] score, 2.7). Serum periostin levels are significantly increased in asthmatic patients with evidence of eosinophilic airway inflammation relative to those with minimal eosinophilic airway inflammation. A logistic regression model, including sex, age, body mass index, IgE levels, blood eosinophil numbers, FENO levels, and serum periostin levels, in 59 patients with severe asthma showed that, of these indices, the serum periostin level was the single best predictor of airway eosinophilia $(\mathrm{P}=.007)$.

Conclusion: Periostin is a systemic biomarker of airway eosinophilia in asthmatic patients and has potential utility in patient selection for emerging asthma therapeutics targeting TH2 inflammation. (Jia et al. ${ }^{11}$, p. 647; Reprinted with permission of Elsevier Ltd.)

2) Comments: Even though asthma was known as mediated by $\mathrm{T}_{\mathrm{H}} 2$ inflammation, there are good evidence of pathophysiologic variability between individuals. Especially in mild to moderate asthma not undergoing steroid treatment, only half of the patients showed evidence of $\mathrm{T}_{\mathrm{H}} 2$ inflammation in their airways. $\mathrm{T}_{\mathrm{H}} 2$ inflammation is characterized by increased markers of allergy, eosinophilic airway inflammation, bronchial fibrosis, and ICS sensitivity. For the evaluation of eosinophilic airway inflammation, sputum eosinophil count or bronchoscopic biopsy, $\mathrm{FE}_{\mathrm{NO}}$ could be used, with different patients' discomfort and cost, resultant variable practicality. There are also several serum markers for $\mathrm{T}_{\mathrm{H}} 2$ inflammation including blood eosinophils, serum IgE, YKL-40, and IL-13. In this study, serum periostin is the only useful biomarker of airway eosinophilic inflammation in asthmatic patients, and it has potential utility in patient selection for on-going asthma therapeutics targeting $\mathrm{T}_{\mathrm{H}} 2$ inflammation without the need for making invasive procedure like bronchoscopic biopsy.

\section{Clinical score systems for severity or prognosis pre- diction}

The asthma disease activity score: a discriminating, responsive measure predicts future asthma attacks. Greenberg et al. ${ }^{12} \mathrm{~J}$ Allergy Clin Immunol 2012;130: 1071-7.

Background: Classifying asthma severity or activity has evolved, but there are no published weighted composite measures of asthma disease activity that account for the relative importance of the many individual clinical variables that are widely used.

Objectives: We sought to develop a weighted and responsive measure of asthma disease activity.

Methods: Discriminant and multiple regression analyses based on 2 previously conducted clinical trials were used to develop 
the Asthma Disease Activity Score (ADAS-6).

Results: The ADAS-6 demonstrated content validity because its components assess different manifestations of asthma: FEV1 (percent predicted), Asthma Quality of Life QuestionnaireSymptom domain, rescue b-agonist use, nocturnal awakenings, peak expiratory flow diurnal variability, and rescue b-agonist use diurnal variability. The ADAS-6 demonstrated crosssectional and longitudinal validity. It was discriminating: it distinguished levels of disease activity and response to different treatment intensities $(P<.0001)$. Similar results were obtained with an independent clinical trial. The ADAS-6 was highly responsive to treatment effects, with a standardized effect size exceeding that of other widely used outcome measures. Using ADAS-6 as the primary end point in the montelukast pivotal trials would have significantly reduced the sample size needed to detect a comparable change in outcome. Furthermore, increments in the ADAS-6 predicted the risk of future asthma attacks. A simplified Asthma Disease Activity Score 4-variable version (ADAS-4) demonstrated similar measurement properties.

Conclusions: The ADAS-6 and ADAS-4 are novel, weighted, and responsive measures of asthma disease activity. Use of these measures in clinical trials might better separate treatment effects, predict future asthma attacks, and substantially reduce sample size. (Greenberg et al. ${ }^{12}$, p. 1071; Reprinted with permission of Elsevier Ltd. )

1) Comments: This study dealt with the derivation of new, composite outcome measures for asthma disease activity, Asthma Disease Activity Score (ADAS) as 6 variable version, ADAS- 6 and 4 variable simplified version, ADAS- 4 . In this study, both versions showed appropriate validity because they include multiple manifestations of asthma itself with adequately moderate internal consistency. Therefore, they might be used as new and trustable measurement system for asthma disease activity in clinical trials and the authors suggest that these scoring systems might significantly reduce sample size needed in each study protocol. Because asthma has multiple, various disease domains including lung function, exacerbation, activity, symptoms control and quality of life, measuring disease activity or predicting disease outcome with only one factor like lung function or symptoms control might results in serious limitation or conclusion. The decision of which from 2 scores to use could depend on the availability of once or twice daily diary data. And, ADAS-6 include 2 more variables, its discriminating ability is better than ADAS-4, the author claimed. In conclusion, when planning important clinical trial in asthma, these scoring systems could provide relatively easy and more practical outcome measurement.

Severity of asthma score predicts clinical outcomes in patients with moderate to severe persistent asthma. Eisner et al. ${ }^{13}$ Chest 2012;141:58-65.
Background: The severity of asthma (SOA) score is based on a validated disease-specifi c questionnaire that addresses frequency of asthma symptoms, use of systemic corticosteroids, use of other asthma medications, and history of hospitalization/ intubation for asthma. SOA does not require measurements of pulmonary function. This study compared the ability of SOA to predict clinical outcomes in the EXCELS (Epidemiological Study of Xolair [omalizumab]: Evaluating Clinical Effectiveness and Long-term Safety in Patients with Moderate to Severe Asthma) patient population vs three other asthma assessment tools. EXCELS is a large, ongoing, observational study of patients with moderate to severe persistent asthma and reactivity to perennial aeroallergens.

Methods: Baseline scores for SOA, asthma control test (ACT), work productivity and impairment index-asthma (WPAI-A), and FEV $1 \%$ predicted were compared for their ability to predict five prespecified adverse clinical outcomes in asthma: serious adverse events (SAEs) reported as exacerbations, SAEs leading to hospitalizations, the incidence of unscheduled offi ce visits, ED visits, and poor IV corticosteroid bursts related to asthma. Logistic regression analysis, area under receiver operating characteristic curves (AUCROCs), and classification and regression tree (CART) analysis were used to evaluate the ability of the four tools to predict adverse clinical outcomes using baseline and 1-year data from 2,878 patients enrolled in the non-omalizumab cohort of EXCELS.

Results: SOA was the only assessment tool contributing significantly in all five statistical models of adverse clinical outcomes by logistic regression analysis (full model AUCROC range, 0.689-0.783). SOA appeared to be a stand-alone predictor for four of fi ve outcomes (reduced model AUCROC range, 0.6890.773). CART analysis showed that SOA had the greatest variable importance for all five outcomes.

Conclusions: SOA score was a powerful predictor of adverse clinical outcomes in moderate to

severe asthma, as evaluated by either logistic regression analysis or CART analysis. (Eisner et al. ${ }^{13}, p .58$; Reprinted with permission of American College of Chest Physicians)

2) Comments: Several scoring systems for asthma severity, disease activity, function impairment, or risk prediction have been established and validated for its usefulness and practicality until now ${ }^{12-14}$. Asthma causes profound morbidity including mild symptoms to exacerbation, there are also issues of evaluation of asthma control and preventing exacerbation ${ }^{13}$. In this study, severity of asthma (SOA) score was a more powerful predictor of poor outcomes in moderate to severe asthma, as evaluated by either logistic regression analysis or classification and regression tree analysis compared to other tools. And, of the assessment tools included and evaluated in this study, SOA score was the only one that was a constituent of full logistic regression model for all the adverse clinical outcomes modeled. These results suggest that composite score 
including clinical symptoms and recent exacerbation could be more important in prediction of clinical course or asthma than lung function only.

\section{Results from GWAS}

Recently, with the results from several GWAS based on large numbered cohort, there have been reports about surrogate genetic markers about susceptibility or prognosis of asthma, and response to certain treatments. If we can detect the clinical prognostic factors based on genetic susceptibility or racial difference, more effective therapeutic strategy could be made to discern highly susceptible individuals even before asthma development, or manage risk groups for severe asthma from the beginnings.

The IL6R variation Asp(358)Ala is a potential modifier of lung function in subjects with asthma. Hawkins et al. ${ }^{15} \mathrm{~J}$ Allergy Clin Immunol 2012;130:510-5.

Background: The IL6R single nucleotide polymorphism (SNP) rs4129267 has recently been identified as an asthma susceptibility locus in subjects of European ancestry but has not been characterized with respect to asthma severity. The SNP rs4129267 is in linkage disequilibrium $(r 2=1)$ with the $I L 6 R$ coding SNP rs2228145 (Asp358Ala). This IL6R coding change increases IL-6 receptor (IL-6R) shedding and promotes IL-6 transsignaling.

Objectives: We sought to evaluate the IL6R SNP rs2228145 with respect to asthma severity phenotypes.

Methods: The IL6R SNP rs2228145 was evaluated in subjects of European ancestry with asthma from the Severe Asthma Research Program (SARP). Lung function associations were replicated in the Collaborative Study on the Genetics of Asthma (CSGA) cohort. Serum soluble IL-6R levels were measured in subjects from SARP. Immunohistochemistry was used to qualitatively evaluate IL-6R protein expression in bronchoalveolar lavage cells and endobronchial biopsies.

Results: The minor C allele of IL6R SNP rs2228145 was associated with a lower percent predicted FEV1 in the SARP cohort $(P=.005)$, the CSGA cohort $(P=.008)$, and in a combined cohort analysis $(P=.003)$. Additional associations with percent predicted forced vital capacity (FVC), FEV1/FVC ratio, and PC20 were observed. The rs2228145 C allele (Ala358) was more frequent in severe asthma phenotypic clusters. Elevated serum soluble IL-6R levels were associated with lower percent predicted FEV1 $(P=.02)$ and lower percent predicted FVC $(P=$ .008) $(\mathrm{n}=146)$. IL-6R protein expression was observed in bronchoalveolar lavage macrophages, airway epithelium, vascular endothelium, and airway smooth muscle.

Conclusions: The IL6R coding SNP rs2228145 (Asp358Ala) is a potential modifier of lung function in subjects with asthma and might identify subjects at risk for more severe asthma.
IL-6 transsignaling might have a pathogenic role in the lung. (Hawkins et al. ${ }^{15}$, p. 510; Reprinted with permission of Elsevier Ltd.)

1) Comments: In this study, the authors have identified the IL6R coding SNP rs2228145 (Asp358Ala) as a potential genetic modifier of lung function in asthma and also as a novel genetic marker of asthma severity. It might identify subjects at risk for more severe asthma or rapid decliner. These observations could be invaluable in the aspect of the recent study direction using GWAS and about 58,000 subjects were included in the recent GWAS that identified $I L 6 R$ as an asthma risk gene. The identification of the IL6R coding SNP rs2228145 (Asp358Ala) as a risk factor for lower lung function showed that dysfunctional IL-6 trans-signaling could be a potential therapeutic target in severe asthma. Therefore, treatment targeting IL-6 trans-signaling could be a novel option for treating several inflammatory diseases, and the results of this study might act as the focus for this therapeutic class of drug in severe asthma. Moreover, genetic test for the IL6R mutation (Asp358Ala), or increased serum sIL-6R levels might be a surrogate marker to identify subjects at risk for more severe asthma, the possible targets of anti-IL-6R therapy.

Genome-wide association study to identify genetic determinants of severe asthma. Wan et al. ${ }^{16}$ Thorax 2012;67:762-8.

Background: The genetic basis for developing asthma has been extensively studied. However, association studies to date have mostly focused on mild to moderate disease and genetic risk factors for severe asthma remain unclear.

Objective: To identify common genetic variants affecting susceptibility to severe asthma.

Methods: A genome-wide association study was undertaken in 933 European ancestry individuals with severe asthma based on Global Initiative for Asthma (GINA) criteria 3 or above and 3346 clean controls. After standard quality control measures, the association of 480,889 genotyped single nucleotide polymorphisms (SNPs) was tested. To improve the resolution of the association signals identified, non-genotyped SNPs were imputed in these regions using a dense reference panel of SNP genotypes from the 1000 Genomes Project. Then replication of SNPs of interest was undertaken in a further 231 cases and 1345 controls and a meta-analysis was performed to combine the results across studies.

Results: An association was confirmed in subjects with severe asthma of loci previously identified for association with mild to moderate asthma. The strongest evidence was seen for the $O R$ $M D L 3 / G S D M B$ locus on chromosome 17q12-21 (rs4794820, $\mathrm{p}=1.03 \times 10(-8)$ following meta-analysis $)$ meeting genome-wide significance. Strong evidence was also found for theIL1RL1/ IL18R1 locus on 2 q12 (rs9807989, p $=5.59 \times 10(-8)$ following 
meta-analysis) just below this threshold. No novel loci for susceptibility to severe asthma met strict criteria for genome-wide significance.

Conclusions: The largest genome-wide association study of severe asthma to date was carried out and strong evidence found for the association of two previously identified asthma susceptibility loci in patients with severe disease. A number of novel regions with suggestive evidence were also identified warranting further study. (Wan et al. ${ }^{16}, p$. 762; Reprinted with permission of BMJ Publishing Group Ltd. )

2) Comments: It was the largest GWAS of severe asthma and also the first GWAS of severe asthma to date. The main question and aim of this study was to identify genetic susceptibility loci to severe asthma and also to evaluate whether there are differences in the genetic susceptibility loci determined by GWAS between severe asthma and mild to moderate asthma. The authors identified several novel loci possibly contribute to the severe asthma, which did not reach the statistical significance level. There might be the possibility that the size of this study population could be smaller than needed for confirmation of the susceptibility, the authors claimed. But, due to the difficulty of recruiting definition-wise severe asthma population more than the size of this study, replication of this study could not be easily performed in the near future. This study also identified that some genetic loci to the development of severe asthma, in which the association with mild to moderate disease was proven. Replication studies for these findings are also warranted.

\section{References}

1. Pavord ID, Korn S, Howarth P, Bleecker ER, Buhl R, Keene ON, et al. Mepolizumab for severe eosinophilic asthma (DREAM): a multicentre, double-blind, placebo-controlled trial. Lancet 2012;380:651-9.

2. Haldar P, Brightling CE, Hargadon B, Gupta S, Monteiro W, Sousa A, et al. Mepolizumab and exacerbations of refractory eosinophilic asthma. N Engl J Med 2009;360:973-84.

3. Brusselle GG, Vanderstichele C, Jordens P, Deman R, Slabbynck $\mathrm{H}$, Ringoet $\mathrm{V}$, et al. Azithromycin for prevention of exacerbations in severe asthma (AZISAST): a multicentre randomised double-blind placebo-controlled trial. Thorax 2013;68:322-9.

4. Albert RK, Connett J, Bailey WC, Casaburi R, Cooper JA Jr, Criner GJ, et al. Azithromycin for prevention of exacerbations of COPD. N Engl J Med 2011;365:689-98.
5. Sutherland ER, King TS, Icitovic N, Ameredes BT, Bleecker E, Boushey HA, et al. A trial of clarithromycin for the treatment of suboptimally controlled asthma. J Allergy Clin Immunol 2010;126:747-53.

6. Kerstjens HA, Engel M, Dahl R, Paggiaro P, Beck E, Vandewalker M, et al. Tiotropium in asthma poorly controlled with standard combination therapy. N Engl J Med 2012;367:1198207.

7. Calhoun WJ, Ameredes BT, King TS, Icitovic N, Bleecker ER, Castro M, et al. Comparison of physician-, biomarker-, and symptom-based strategies for adjustment of inhaled corticosteroid therapy in adults with asthma: the BASALT randomized controlled trial. JAMA 2012;308:987-97.

8. Brozek JL, Kraft M, Krishnan JA, Cloutier MM, Lazarus SC, Li JT, et al. Long-acting beta2-agonist step-off in patients with controlled asthma. Arch Intern Med 2012;172:1365-75.

9. Wells KE, Peterson EL, Ahmedani BK, Severson RK, GleasonComstock J, Williams LK. The relationship between combination inhaled corticosteroid and long-acting beta-agonist use and severe asthma exacerbations in a diverse population. J Allergy Clin Immunol 2012;129:1274-9.e2.

10. Sumino K, Sugar EA, Irvin CG, Kaminsky DA, Shade D, Wei $\mathrm{CY}$, et al. Methacholine challenge test: diagnostic characteristics in asthmatic patients receiving controller medications. J Allergy Clin Immunol 2012;130:69-75.e6.

11. Jia G, Erickson RW, Choy DF, Mosesova S, Wu LC, Solberg OD, et al. Periostin is a systemic biomarker of eosinophilic airway inflammation in asthmatic patients. J Allergy Clin Immunol 2012;130:647-54.e10.

12. Greenberg S, Liu N, Kaur A, Lakshminarayanan M, Zhou Y, Nelsen L, et al. The asthma disease activity score: a discriminating, responsive measure predicts future asthma attacks. J Allergy Clin Immunol 2012;130:1071-7.e10.

13. Eisner MD, Yegin A, Trzaskoma B. Severity of asthma score predicts clinical outcomes in patients with moderate to severe persistent asthma. Chest 2012;141:58-65.

14. Schatz M, Zeiger RS, Yang SJ, Chen W, Crawford W, Sajjan S, et al. The relationship of asthma impairment determined by psychometric tools to future asthma exacerbations. Chest 2012;141:66-72.

15. Hawkins GA, Robinson MB, Hastie AT, Li X, Li H, Moore WC, et al. The IL6R variation Asp(358)Ala is a potential modifier of lung function in subjects with asthma. J Allergy Clin Immunol 2012;130:510-5.e1.

16. Wan YI, Shrine NR, Soler Artigas M, Wain LV, Blakey JD, Moffatt MF, et al. Genome-wide association study to identify genetic determinants of severe asthma. Thorax 2012;67:762-8. 